\title{
SYNCHRONOUS MULTIPLE PRIMARY MALIGNANT NEOPLASMS: A CASE REPORT OF MALIGNANT PERITONEAL MESOTHELIOMA AND NEUROENDOCRINE RECTAL TUMOR
}

\author{
Oleksandr Bondar \\ Department of Radiation Diagnostics, Therapy and Oncology \\ Center of Reconstructive and Restorative Medicine (University Clinic) ${ }^{l}$ \\ Sergii Chetverikov \\ Department of Surgery No. 3 \\ Center of Reconstructive and Restorative Medicine (University Clinic) ${ }^{1}$ \\ Viacheslav Maksymovskyi \\ Department of Surgery No. 3 \\ Center of Reconstructive and Restorative Medicine (University Clinic) ${ }^{1}$ \\ Dmytro Atanasov \\ Department of Surgery No. 3 \\ Center of Reconstructive and Restorative Medicine (University Clinic) ${ }^{1}$ \\ Mykhailo Chetverikov \\ Center of Reconstructive and Restorative Medicine (University Clinic) ${ }^{l}$ \\ Valeriia Chetverikova-Ovchynnyk $\bowtie$ \\ Center of Reconstructive and Restorative Medicine (University Clinic) ${ }^{l}$ \\ ovchinnik.val@yahoo.com \\ ${ }^{1}$ Odessa National Medical University \\ 2 Valikhovskyi lane, Odessa, Ukraine, 65082
}

$\triangle$ Corresponding author

\begin{abstract}
The aim of the research was to discuss a rare case of synchronous malignant peritoneal mesothelioma of the biphasic histological type and neuroendocrine tumor (NET) of the rectum without history of asbestos exposure and to analyze the outcomes of the performed treatment.

Materials and methods. The 48-year-old patient with synchronous malignant peritoneal mesothelioma of the biphasic histological type and neuroendocrine tumor (NET) of the rectum was analyzed for oncological and clinical outcomes.

Case presentation. During 2 years since manifestation of the disease the patient underwent 3 cytoreductive surgeries (CRS): removal of the tumor of the sigmoid mesentery, resection of the rectosigmoid junction completeness of cytoreduction (CC) 0 (2017), omentectomy and partial parietal peritonectomy CC-0 (2017), atypical resection of S2, S4, S5 liver, the removal of the abdominal tumor with left-sided en-block hemicolectomy, partial parietal peritonectomy, argon-plasma coagulation of tumor foci on the mesentery of the small intestine CC-2 (2018) and Transanal Minimally Invasive Surgery-removal of neuroendocrine rectal tumor (2017). The patient underwent hyperthermic intraperitoneal chemotherapy (HIPEC) twice (during $2^{\text {nd }}$ and $3^{\text {rd }} \mathrm{CRS}$ ). Different regimens of HIPEC were performed: cisplatin + doxorubicin (2017) and metamycin C (2018). The patient received 4 courses of adjuvant chemotherapy with cisplatin plus pemetrexed in 2017 and 3 courses of the chemotherapy with gemcitabine and carboplatin plus bevacizumab in 2018.

Results. The patient survived 21 months after the detection of malignant peritoneal mesothelioma in 2017 and died 4 months after the last cytoreductive surgery from the progression of the disease.

Conclusions. Histological subtype of MPMP remains important factor in the prognosis of the disease even on the early stages though patient had received the most aggressive variant of special treatment. Minimally invasive treatment tactics of NET demonstrated clinical effectiveness.
\end{abstract}

Keywords: peritoneal mesothelioma, neuroendocrine tumor, hyperthermic intraperitoneal chemotherapy, cytoreductive surgery. 


\section{Introduction}

Since the 1990s, the techniques for cancer diagnostics and treatment have greatly improved worldwide, which have increased the survival period of cancer patients. The prolonged overall survival of cancer patients also led to an increase in the incidence of multiple primary malignant tumors (two or more histologically distinct malignant tumors that appeared not due to recurrence, metastasis or local spread in the same individual). The first report about multiple primary malignant neoplasms in a single patient was published at the end of the 19th century [1].

Since then, several papers worldwide have addressed this issue and the prevalence of multiple primary malignant neoplasms reported varies from $0.734 \%$ to $11.7 \%$ [2]. Multiple neoplasms could be defined by when they occur as synchronous and metachronous. The co-existence of malignant peritoneal mesothelioma and neuroendocrine tumor of the rectum is a rare entity.

Malignant mesothelioma is a rare disease that develops from multipotent mesothelial cells of serosal surfaces comprising the pleura, peritoneum, pericardium and tunica vaginalis testis. The incidence of diffuse malignant peritoneal mesothelioma (MPMP) reaches $7-30 \%$ of all cases [3]. The highest rates are reported in the UK, Australia, and New Zealand. An estimated annual incidence of MPM is 2500 cases worldwide [4]. Neuroendocrine tumors originate from neuroendocrine cells of endodermal origin, spreading along the gastrointestinal tract. Prevalence of neuroendocrine tumor is around 35 cases per 100,000 including all types [5].

Patients with neuroendocrine tumors (NET) of the colon and rectum have a high risk of developing metachronous non-carcinoid malignant tumors including malignant mesothelioma with a frequency of 25-46\% [6]. Multiple tumors in these cases have a higher potential for malignancy, therefore, patients have progression of the disease.

NETs of rectal origin almost never present with the traditional «carcinoid syndrome» symptoms characterized by episodic cutaneous flushing and diarrhea [7]. They are often asymptomatic, but approximately $50 \%$ can present with rectal bleeding, changes in bowel habits, unexplained weight loss, or pain.

In most cases, the clinical presentation of MPM is also asymptomatic. There are no pathognomonic symptoms at initial stages. According to a multicentre cohort study the most frequent symptoms/signs were ascites (77\%), abdominal pain (69\%), asthenia (43\%), weight loss (32\%), abdominal swelling (30\%) and loss of the appetite (30\%) [3]. Due to the nonspecific nature and late manifestation of these symptoms, the decease is often diagnosed at the advanced stages. The median time from clinical manifestation to the diagnosis is about 4 months [8].

Current guidelines suggest endoscopic resection of rectal NETs $\leq 10 \mathrm{~mm}$ as a safe therapeutic option because of their low risk of metastasis [7].

MPM is a life-threatening malignancy that, with no treatment, has a median overall survival of 8.4 months [9]. Cytoreductive surgery (CRS) with hyperthermic intraperitoneal chemotherapy (HIPEC) has become the consensus standard first-line therapy for MPM [3]. The median overall survival after CRS + HIPEC ranged from 15 to 92 months $[3,4,9]$. This wide range apparently reflects various surgeons experience, different status of patients and no standardized technique for HIPEC.

Herein, we report a rare case of synchronous malignant peritoneal mesothelioma and neuroendocrine tumor of the rectum without history of asbestos exposure.

The aim of the research was to discuss a case of synchronous malignant peritoneal mesothelioma of the biphasic histological type and neuroendocrine tumor (NET) of the rectum without history of asbestos exposure and to analyze the outcomes of the performed treatment.

\section{Materials and methods}

The 48-year-old patient with synchronous malignant peritoneal mesothelioma of the biphasic histological type and neuroendocrine tumor (NET) of the rectum was analyzed for oncological and clinical outcomes. Clinical, laboratory and instrumental examination of patient was performed according to the recommendations of the Peritoneal Surface Oncology Group International/Rare Adult Solid Cancer clinical practice guidelines for diagnosis, treatment and follow-up of peritoneal mesothelioma. The tumor was verified using histological and immunohistochemical examinations. Cytoreductive surgery, HIPEC, transanal minimal invasive surgery, adjuvant chemotherapy were 
performed. The condition of patient was assessed by analyzing clinical, instrumental and laboratory parameters.

\section{Presentation of the case}

The research was carried out within the framework of the scientific work of the Department of radiation diagnostics, treatment and oncology of the Odessa National Medical University. Compliance with the WMA Declaration of Helsinki - Ethical Principles for Medical Research Involving Human Subjects, 2013 (minutes of the meeting of the Committee on Bioethics of the Odessa National Medical University No. 148A dated June 15, 2019) was determined. Study participant was informed and agreed to the processing of his clinical data and to participate in the research process.

Patient V., 48 years old, consulted his family physician in May 2017 with complaints of low-grade fever, dry cough and weight loss during the last 2 months. Computed tomography with intravenous contrast (CT) of the chest and the abdomen was performed. According to the CT of the abdomen, there was a tumor of the sigmoid mesentery with a diameter of up to $7 \mathrm{~cm}$. In July 2017 he was admitted to the surgery department of the oncological center. The removal of the tumor of the sigmoid mesentery, resection of the rectosigmoid junction was performed. Intraoperative peritoneal cancer index (PCI) was estimated 3 points. According to the first histological description, the tumor was undifferentiated pleiomorphic sarcoma. Immunohistochemical study was performed and malignant mesothelioma of the biphasic type was verified. The patient consulted a clinical oncologist in August 2017. Due to the stage 1 disease, no special postoperative treatment was indicated.

In August 2017, according to video-colonoscopy with a biopsy, a submucous tumor of the mid-ampullar rectum was found, histologically there were revealed the elements of carcinoma. Transanal Minimally Invasive Surgery (TAMIS)-removal of a submucous tumor of the mid-ampullar rectum was performed. Histologically and immunohistochemicaly neuroendocrine carcinoma pT1N0M0G1, pSt.I grade 1 malignancy (atypical carcinoid) was verified.

In September 2017, a screening CT of the chest and the abdomen was performed. CT signs of peritoneal carcinomatosis were described. The serum levels of the tumor markers (carcinoembryonic antigen (CEA) and carbohydrate antigen 125 (CA125)) were not elevated.

Considering the CT signs of peritoneal mesothelioma recurrence the patient was admitted to the oncological center. Laparoscopic staging and the biopsy of the parietal peritoneum were performed. PCI was estimated 4 points. Cytoreductive surgery and HIPEC were recommended to perform.

In November 2017, patient V. was admitted to the University Clinics of Odessa National Medical University. Intraoperative PCI was estimated 5 points. The macroscopically complete cytoreductive (CC-0) was achieved, which included omentectomy and partial parietal peritonectomy. HIPEC with cisplatin $100 \mathrm{mg}$, doxorubicin $30 \mathrm{mg}$ was performed using the «closed abdomen» technique $\left(41^{\circ} \mathrm{C}, 90^{\prime}\right)$. According to the results of histological examination, malignant mesothelioma of the biphasic type was verified. The patient was discharged 7 days after CRS + HIPEC in a stable condition.

During the next 3 months, the patient received 4 courses of adjuvant chemotherapy with cisplatin plus pemetrexed (PC).

According to the screening CT of the chest, abdomen and pelvis in February 2018, a recurrent tumor was detected in the area of the left colic flexure. The patient refused the surgical treatment.

From February to May 2018 he received 3 courses of the chemotherapy with gemcitabine and carboplatin (GC) plus bevacizumab.

According to the control CT of the chest, abdomen and pelvis in May and August 2018, the enlargement of the tumor size up to $8 \mathrm{~cm}$.

Since September 2018 the patient had concerned on constant fever, pain in the left hypochondrium and decrease in body weight. According to the blood test, there was a decrease in the level of hemoglobin. In September the CT imaging confirmed multiple nodular tumors of the liver suspected to be metastatic (CT of the chest, abdomen, retroperitoneal space and pelvis 09/20/2018 - Disease progression. Tumors S2, S4, S5, S8 of the liver up to $1 \mathrm{~cm}$ in diameter, probably metastatic are determined. Partially adhering to the splenic angle of the colon, partly to the abdominal wall, a multicameral extraorganic cystic and solid tumor of irregular shape $103 \times 41 \times 67 \mathrm{~mm}$ in size is determined). According to the data of control video-colonoscopy, no signs of tumor growth were revealed. 
In October 2018 percutaneous ultrasound guided liver biopsy was performed. According to the results of the histological and immunohistochemical examination, the material is dominated by liver tissue with a focus of malignant tumor: carcinoma with loss of receptor status after chemotherapy. Taking into account the presence of intractable pain, hyperthermic syndrome, sub-compensated bowel obstruction, no positive effect of the systemic chemotherapy, CRS and HIPEC was recommended.

In November 2018 he was admitted to the University Clinics of Odessa National Medical University for further examination and treatment. At the time of hospitalization, he complained about constant pain in the left hypochondriac and left lateral region, which aggravated during inspiration and cough; febrile fever, night sweats, dry cough at the height of fever, general weakness, hyporexia, rapid breathing, weight loss up to $10 \mathrm{~kg}$ in the last 2 months, constipation, hard stools, dizziness, shortness of breath, palpitations. Taking paracetamol, ketorolac was without positive effect.

Intraoperative PCI was estimated 21 points. During the revision of the abdomen metastatic lesion of the liver, tumor invasion of the splenic flexure of the colon, peritoneal carcinomatosis of the parietal and visceral peritoneum were verified. Suboptimal cytoreductive surgery (CC-2) was performed. The extent of the surgery was laparotomy, atypical resection of S2, S4, S5 liver, the removal of the abdominal tumor with left-sided en-block hemicolectomy, partial parietal peritonectomy, argon-plasma coagulation of tumor foci on the small intestinal mesentery. Intra-abdominal hyperthermic chemotherapy with metamycin $\mathrm{C}$ was performed using the «closed abdomen» technique. The surgery was sub-optimal due to the large extent of the disease and unresectable liver metastasis. Intraoperative transfusion of isogroup erythrocyte mass of $600 \mathrm{ml}$ was performed, transferred without post-transfusion reactions, complications.

The patient was discharged on the 14th postoperative day in a stable condition, the paraneoplastic syndrome was arrested.

Further adjuvant palliative polychemotherapy was not given because the patient refused additional therapy.

Based on CT screening data and clinically, disease progression was observed in February 2019. The patient survived 21 months after the detection of malignant peritoneal mesothelioma in 2017 and died 4 months after the last cytoreductive surgery from the progression of the disease.

\section{Result and discussion}

Based on literature data, rectal NETs compose 1-2\% of all rectal tumors, and generally demonstrate a benign clinical profile [7]. After minimally invasive tumor resection, patient V. did not receive progression or recurrence of rectal neuroendocrine carcinoma according to video-colonoscopy and CT. In this case, the chosen treatment tactics has shown its clinical effectiveness.

According to the review of Kim J. et al. Without treatment, patients with MPMP have a median survival time of under 5 months to a year after diagnosis [8].

For MPMP, the histological subtype has a decisive influence on the life expectancy of patients, regardless of the specific treatment performed. In the research of Amin et al., for epithelioid histological type the median survival reaches 18 months (95\% CI: 17-21) as compared to 10 months for biphasic (95\% CI: 9-13) and 7 months for sarcomatoid subtype (95\%CI: 6-11) [10]. The research included patients, who received surgery alone, surgery combined with chemotherapy and best supportive care. Also, it has been suggested that female patients with mesothelioma have a better life expectancy as compared to male patients.

Therefore, it is important to confirm MPMP and to define its histological subtype before consider CRS/HIPEC. Patients with the sarcomatoid variant of MPMP are not candidates for CRS; however, epithelioid and highly selected biphasic cases are.

According to the Kim J. et al. investigation, patients with biphasic subtype do not have the same survival benefit from CRS+HIPEC when compared to those with epithelioid subtype. In these patients, the decision for CRS-HIPEC or systemic chemotherapy is more debatable [8].

In our clinical case, patient received benefit in overall survival compared to the data of the literature (21 vs 10 months). The patient had received HIPEC twice (in 2017 with cisplatin + doxo- 
rubicin and nearly 12 months later with metamycin C). Both of these chemoperfusion regimens are currently used for treatment of MPMP [11].

The most effective cytostatic therapy for MPMP is considered pemetrexed, with either cisplatin or carboplatin. Failure to obtain disease control is an indication for another regimen such as cisplatin with irinotecan, gemcitabine with cisplatin/carboplatin [12]. Another alternative for second-line systemic treatment is the use of a molecular agent, such as bevacizumab [12]. All of the second-line therapies are still under investigation.

Study limitations. The limitations were the lack of ability to generalize, no possibility to establish cause-effect relationship, danger of over-interpretation, publication bias and the retrospective design of the study.

Prospects for further research. The research should be continued in a larger number of patients to form the effective approaches to the treatment and diagnosis of malignant peritoneal mesothelioma.

\section{Conclusions}

Both MPMP and NET of the rectum could be diagnosed on the late stages due to their asymptomatic clinics. MPMP could be misdiagnosed as recurrence or metastasis of another malignancy in patient with synchronous or metachronous malignancies, which may result in inappropriate treatment. This inevitably could have negative influence on the patient's prognosis.

Malignant peritoneal mesothelioma creates a challenge for oncologists in terms of rare silent disease, diagnosis, complex treatment and poor prognosis. MPMP continues to have a poor prognosis because of the limited therapeutic options. Because of the rarity of disease, it is difficult to provide patient participation in prospective clinical trials with the purpose of treatment standardization. Histological subtype of the tumor remains an important factor in the prognosis of the disease even on the early stages though patient had received the most aggressive variant of special treatment.

\section{Conflict of interest}

The authors declare that they have no conflicts of interest.

\section{Financing}

The study was performed without financial support.

\section{Acknowledgments}

We would like to express our gratitude to the administration of the Odessa national medical university who agreed to conduct the study.

\section{References}

[1] Zhai, C., Cai, Y., Lou, F., Liu, Z., Xie, J., Zhou, X. et. al. (2018). Multiple Primary Malignant Tumors - A Clinical Analysis of 15,321 Patients with Malignancies at a Single Center in China. Journal of Cancer, 9 (16), 2795-2801. doi: http://doi.org/ $10.7150 /$ jca. 25482

[2] Ozbudak, I. H., Ozbudak, O., Arslan, G., Erdogan, A., Ozbilim, G. (2013). Metachronous malignant mesothelioma and pulmonary adenocarcinoma. Turkish Journal of Pathology, 29 (1), 83-86. doi: http://doi.org/10.5146/tjpath.2013.01156

[3] Kusamura, S., Kepenekian, V., Villeneuve, L., Lurvink, R. J., Govaerts, K., De Hingh, I. H. J. T. et. al. (2021). Peritoneal mesothelioma: PSOGI/EURACAN clinical practice guidelines for diagnosis, treatment and follow-up. European Journal of Surgical Oncology, 47 (1), 36-59. doi: http://doi.org/10.1016/j.ejso.2020.02.011

[4] Boussios, S., Moschetta, M., Karathanasi, A., Tsiouris, A. K., Kanellos, F. S., Tatsi, K. et. al. (2018). Malignant peritoneal mesothelioma: clinical aspects, and therapeutic perspectives. Annals of gastroenterology, 31 (6), 659-669. doi: http://doi.org/ 10.20524/aog.2018.0305Rakici, H., Akdogan, R. A., Yurdakul, C., Canturk, N. (2015). A case of rectal neuroendocrine tumor presenting as polyp. International Journal of Surgery Case Reports, 8, 59-61. doi: http://doi.org/10.1016/j.ijscr.2015.01.031

[5] Scherübl, H. (2011). Management of early gastrointestinal neuroendocrine neoplasms. World Journal of Gastrointestinal Endoscopy, 3 (7), 133-139. doi: http://doi.org/10.4253/wjge.v3.i7.133

[6] Chablaney, S., Zator, Z. A., Kumta, N. A. (2017). Diagnosis and Management of Rectal Neuroendocrine Tumors. Clinical Endoscopy, 50 (6), 530-536. doi: http://doi.org/10.5946/ce.2017.134 
[7] Kim, J., Bhagwandin, S., Labow, D. M. (2017). Malignant peritoneal mesothelioma: a review. Annals of Translational Medicine, 5 (11), 236-236. doi: http://doi.org/10.21037/atm.2017.03.96

[8] Marques de Sousa, S., Pereira, F., Duarte, M., Marques, M., Vázquez, D., Marques, C. (2019). Malignant Peritoneal Mesothelioma as a Rare Cause of Dyspeptic Complaints and Ascites: A Diagnostic Challenge. GE - Portuguese Journal of Gastroenterology, 27 (3), 197-202. doi: http://doi.org/10.1159/000503075

[9] Amin, W., Linkov, F., Landsittel, D. P., Silverstein, J. C., Bshara, W., Gaudioso, C. et. al. (2019). Factors influencing malignant mesothelioma survival: a retrospective review of the National Mesothelioma Virtual Bank cohort. F1000Research, 7, 1184. doi: http://doi.org/10.12688/f1000research.15512.3

[10] Enomoto, L. M., Shen, P., Levine, E. A., Votanopoulos, K. I. (2019). Cytoreductive surgery with hyperthermic intraperitoneal chemotherapy for peritoneal mesothelioma: patient selection and special considerations. Cancer Management and Research, 11, 4231-4241. doi: http://doi.org/10.2147/cmar.s170300

[11] Broeckx, G., Pauwels, P. (2018). Malignant peritoneal mesothelioma: a review. Translational Lung Cancer Research, 7 (5), 537-542. doi: http://doi.org/10.21037/tlcr.2018.10.04

Received date 21.06.2021

(C) The Author(s) 2021

Accepted date 27.07.2021

Published date 30.07.2021

This is an open access article under the Creative Commons CC BY license

How to cite: Bondar, O., Chetverikov, S., Maksymovskyi, V., Atanasov, D., Chetverikov, M., Chetverikova-Ovchynnyk, V. (2021). Synchronous multiple primary malignant neoplasms: a case report of malignant peritoneal mesothelioma and neuroendocrine rectal tumor. EUREKA: Health Sciences, 4, 81-86. doi: http://doi.org/10.21303/2504-5679.2021.001898 